\title{
Curvature-Scale-based Contour Understanding for Leaf Margin Shape Recognition and Species Identification*
}

\author{
Guillaume Cerutti ${ }^{1,2}$, Laure Tougne ${ }^{1,2}$, Didier Coquin ${ }^{3}$ and Antoine Vacavant ${ }^{4}$ \\ ${ }^{1}$ Université de Lyon, CNRS \\ ${ }^{2}$ Université Lyon 2, LIRIS, UMR5205, F-69676, France \\ ${ }^{3}$ LISTIC, Domaine Universitaire, F-74944, Annecy le Vieux \\ ${ }^{4}$ Clermont Université, Université d'Auvergne, ISIT, F-63001, Clermont-Ferrand \\ \{guillaume.cerutti, laure.tougne\}@liris.cnrs.fr, didier.coquin@univ-savoie.fr, antoine.vacavant@iut.u-clermont1.fr
}

\begin{abstract}
Keywords: curvature-scale space, contour characterization, leaf identification, shape classification
Abstract: In the frame of a tree species identifying mobile application, designed for a wide scope of users, and with didactic purposes, we developed a method based on the computation of explicit leaf shape descriptors inspired by the criteria used in botany. This paper focuses on the characterization of the leaf contour, the extraction of its properties, and its description using botanical terms. Contour properties are investigated using the Curvature-Scale Space representation, the potential teeth explicitly extracted and described, and the margin classified into a set of inferred shape classes. Results are presented for both margin shape characterization, and leaf classification over nearly 80 tree species.
\end{abstract}

\section{INTRODUCTION}

Plants, trees and herbs that used to constitute the most immediate environment for past generations, seem somehow disconnected from our everyday life, in a world of rampant urbanization and invasive technology. Knowledge of the uses and properties of numerous species got away, kept only by a handful of botanists. Even identifying a simple plant has merely become a case for the specialists.

But the blossoming of mobile technology curiously offers the opportunity of spreading back this knowledge into everyone's pocket. Providing an intuitive and flexible way to recognize species, to teach anyone who feels the need how to look at a plant, is now a possibility. Attempts in this direction have come to light with great success, being with user-based (TreeId, Fleurs en Poche) or automatic recognition (LeafSnap ${ }^{1}$ ) on white background images.

Leaves are a choice target for such application, present almost all year long, easy to photograph, and

\footnotetext{
${ }^{*}$ This work has been supported by the French National Agency for Research with the reference ANR-10-CORD005 (REVES project).

${ }^{1} \mathrm{http}: / /$ leafsnap.com : developed by researchers from Columbia University, the University of Maryland, and the Smithsonian Institution (Belhumeur et al., 2008)
}

with well studied geometrical specificities that make the identification, if not trivial, possible. Our main objective is to build a system for leaf shape analysis of photographs in a natural environment, relying on high-level geometric criteria inspired by those used by botanists to classify a leaf into a list of species.

In this paper, we focus on the characterization of the leaf margin shape, introducing a dedicated description of the shape contour. Section 2 presents works connected to this matter. The processing performed on the contour is described in Section 3 and the descriptor we use detailed in Section 4. Section 5 expounds its interest for both margin shape classification and species identification, and conclusions are drawn in Section 6.

\section{RELATED WORKS}

\subsection{Leaf Identification}

Leaf image retrieval and plant identification have been a growing topic of interest in the past few years. Some authors (Belhumeur et al., 2008) also aim at conceiving a mobile guide, achieving great performance on plain background images by the combination of established shape descriptors (InnerDistance Shape Context) and classification methods. 
Some works tackle the challenge of segmentation over natural background (Teng et al., 2009; Wang et al., 2008), but most elude this obstacle, working on plain background images, where sensitivity to noisy shapes is less of an issue given the general accuracy of the obtained contours.

Many methods rely on statistical features such as moments (Wang et al., 2008), histogram of gradients or local interest points (Goëau et al., 2011b) or generic contout descriptors such as the CurvatureScale Space (Mokhtarian and Abbasi, 2004). Such descriptors were not designed to take into account the nature of the object, but fit quite well with its specificities. A commonly used geometrical descriptor for leaf image retrieval is the CentroidContour Distance (CCD) curve (Wang et al., 2000; Teng et al., 2009) though it can be applied to any type of object.

On the other hand, some morphological features explicitly computed on the shape of the object to model its natural properties have also been used ( $\mathrm{Du}$ et al., 2007; Goëau et al., 2011b). Even more dedicated methods have been designed, basing their recognition on an explicit representation of the leaf contour and of its teeth (Im et al., 1998; Caballero and Aranda, 2010) but suitable only for image retrieval and applied to a small number of species.

\subsection{Curvature for Contour Description}

Since the leaf margin shape is a very discriminant feature for identification, characterizing the contour of the leaf is a crucial step. The CCD curve provides an interesting view, but lacks precision, as the curve for a leaf with small teeth will be very close to a smooth one. To discriminate such contours, the use of curvature is very advantageous.

A rich representation of the contour is the Curvature-Scale Space (CSS), that has already been used in the context of shape recognition (Mokhtarian and Mackworth, 1992) and even leaf image retrieval (Mokhtarian and Abbasi, 2004; Caballero and Aranda, 2010). It piles up curvature measures at each point of the contour over successive smoothing scales, summing up the information into a map where concavities and convexities clearly appear.

Curvature has also been used to detect dominant points on the contour, and provide a compact description of a contour by its curvature optima. This is a well studied problem (Teh and Chin, 1989) in which the introduction of the curvature-scale transform has proved to be beneficial (Pei and Lin, 1992). The detection and characterization of salient features on a leaf contour is a problem that can be addressed with a similar perspective.

\section{CONTOUR INTERPRETATION}

Our starting point is a segmented leaf, from either a plain or natural background, along with its preliminary global shape estimation (Cerutti et al., 2011). This polygonal model conveys interesting information on the leaf's geometry, and provides notably the number of appearing lobes $n_{L}$ and an estimation of its main axis (possibly more than one in the case of a palmately lobed leaf). Our frame of work here comprises only simple and palmately lobed leaves (nearly $75 \%$ of all European tree specie) species with compound leaves being left aside. Since our goal is to represent the morphology of the margin, knowing where to look is important to select the accurate features and, for instance, not consider the apex as a simple tooth.

\subsection{Leaf Contour Partition}

The contour needs then to be divided into areas corresponding to the apex, to the base, to the potential lobe tips, and finally to the rest of the margin, so that the most salient features do not absorb the rest of the information. Relying on the polygonal leaf model is here useful to solve what would otherwise be a much more complicated task. Supposing that the axes of the polygonal model are accurate, we can use them to mark off contour points that are in one of these areas of interest.
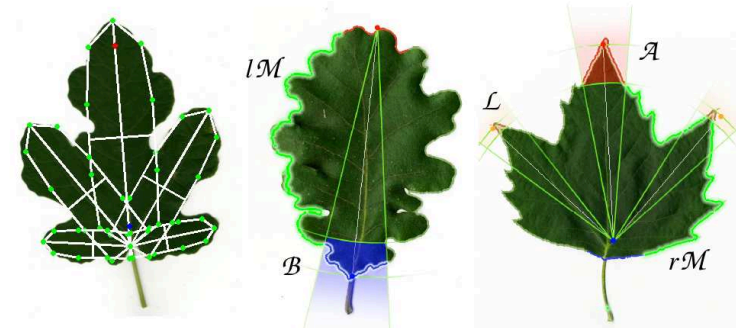

Figure 1: Polygonal model and labelling of contour parts corresponding to leaf areas

To achieve the labelling shown in Figure 1, we define sets of vertices as the intersection of the contour with a fixed angular sector, built around the corresponding axis using the model points, issuing from the opposite point (base for the apices, and apex for the base) and with a minimal distance relatively to this point: $\mathcal{A}$ for the apical area (red), $\mathcal{B}$ for the basal area (blue), $\mathcal{L}$ for the lobe tips (brown), and the actual margin area $\mathcal{M}$, divided into for $l \mathcal{M}$ the left margin area and $r \mathcal{M}$ for the left margin area (green).

This partition first makes possible the accurate location of the actual base point $\hat{B}$ and the apex point $\hat{A}$ of the leaf, as the most salient features in 
their respective area $\mathcal{B}$ or $\mathcal{A}$, a crucial information to estimate the local shape of the leaf around them, a determining feature for botanists. And secondly, outside of these areas, we can study the properties of potential teeth, lobes, sinuses and other salient features in $\mathcal{M}$, and characterize the leaf margin shape, and only it.

\subsection{Curvature-Scale Space Transform}

As a matter of fact, the salient features on the contour appear very clearly on the Curvature-Scale Space transform of the contour, as shown in Figure 3(b). The CSS is a very powerful description but is too informative to be used as a descriptor and to build class models by averaging several of them. As we wish to locate and characterize precisely curvaturedefined elements on the contour, it was a more judicious choice to look, not at zero-curvature points as the original method would do (Mokhtarian and Mackworth, 1992), but on the contrary at the maxima and minima of curvature. These salient points are the ones that visually stand out in the CSS image and can be intuitively be matched to existing structures on the leaf contour, reason why we want to detect them explicitly.

\subsection{Detecting Teeth and Pits}

To locate these points, we actually want to detect dominant points on the leaf contour and to find the scale of the concave or convex part they correspond to. The method we use is largely inspired of existing works (Teh and Chin, 1989; Pei and Lin, 1992) where the process of detection is performed at each scale level. Starting with $S=4$ (lower scale elements being arguably indiscernible from noise) the set of dominant points at each scale $D(S)$ is initialized with all the contour points and the process is then the following:

1. Extract points for which the curvature value is a local optimum in a neighbourhood of size $S$

2. Suppress points the curvature intensity of which is below a threshold $k_{\min }$

3. Suppress points that can not be traced to a dominant point at the previous scale (if $S>4$ )

4. Keep only the median point of potential remaining groups of size $\leq S$

The result we obtain is a map of the located dominant points at each scale, as presented in Figure 3(c). This representation reveals the teeth and pits of the contour in the form of chains of dominant points.

\section{LEAF MARGIN DESCRIPTION}

Once the salient features are detected on the contour, it is necessary to interpret this dense information in order to make the discriminant characteristics of the margin appear. To know what to look for, we relied on the distinctions made by botanists to discriminate the different species.

\subsection{Leaf Margin Shapes in Botany}

To describe the shape of the leaf margin, botanists use a terminology that refers both to the properties of teeth taken separately and to their repartition over the margin. Some of these terms are shown in Figure 2. For instance a "doubly serrate" leaf implies two levels of teeth with different sizes and frequencies, with bigger teeth divided into smaller sub-teeth. The words themselves are vague enough so that leaves with teeth that look rather different may fall inside the same term.
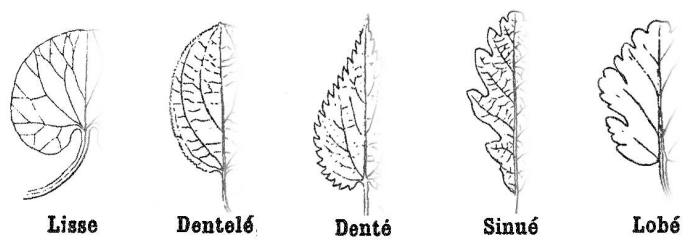

Figure 2: Examples of leaf margin shapes : Entire, Denticulate, Dentate, Sinuate, Lobate. Images taken from (Coste, 1906)

The margin shape constitutes however a very discriminant criterion for species identification that generally presents less variability than the global shape, with the exception of some pathological species. Our descriptor will have to capture the differences regarding size, sharpness, orientation, repartition and variability of the teeth implied in the botanical terms, but also to benefit from the use of numerical values to be more precise and discriminate leaves of different aspect that would be called the same.

\subsection{Margin Interpretation}

For the sake of interpretation, we want to use the representation of the contour by dominant points to retrieve the properties of every located structure on the contour. Each chain that appears on the dominant points map corresponds indeed to a convex or a concave part, and its actual size, as a human eye would interpret it, is the scale until which it persists, that is to say the scale of the chain's end point. 


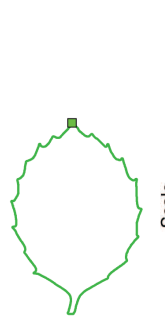

(a)

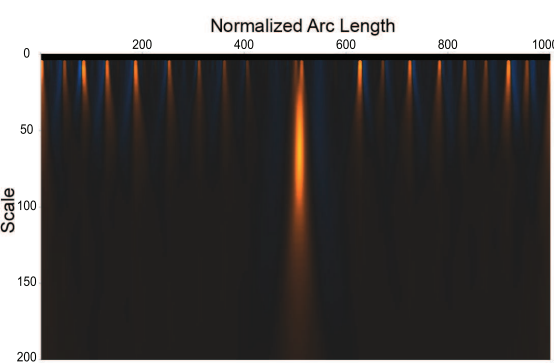

(b)

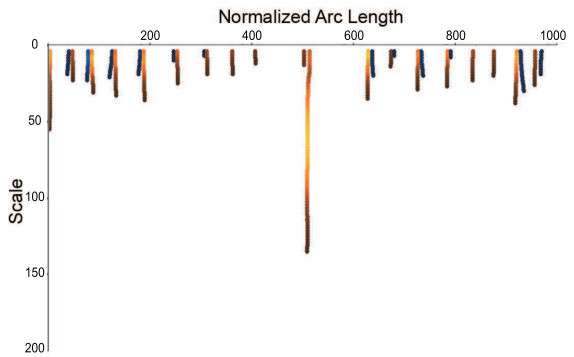

(c)

Figure 3: Traced dominant points over scale (c) and their curvature, obtained from the curvature-scale space transform (b) of a leaf contour (a)

We scan the dominant points starting from the highest scale to keep only these terminal points. When a dominant point is found at scale $S$, all the dominant points of the same curvature sign are simply suppressed at lower scales, in a neighbourhood of size $S$. This way, we ensure that a single structure is not counted twice, and small structures which are included in larger ones are merged into one.

Each point kept after this selection step corresponds to a concave or convex structure of scale $S$. To complete this size information, we estimate the actual curvature $\bar{K}$ of a dominant point of scale $S$ belonging to $D(S)$, as the average curvature of all the dominant points belonging to the same chain. This computation is done while suppressing the points at lower scales.

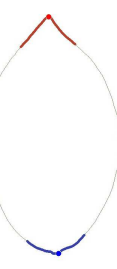

(a)

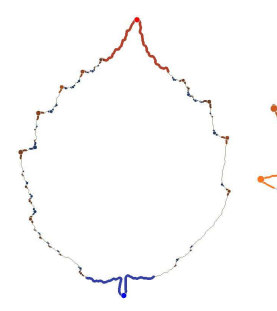

(b)

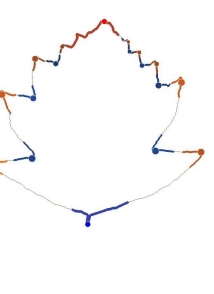

(c)
Figure 4: Various leaf contours with detected base, apex, teeth and pits ; apex area in red, base area in dark blue ; convexities in orange, concavities in blue, brightness representing curvature intensity, extent representing scale.

Finally the final set of dominant points is a semantically rich interpretation of the leaf contour, where the base and apex points $\hat{B}$ and $\hat{A}$ are precisely located, and where teeth are detected and characterized independently in terms of position on the contour $(u)$, size $(S)$ and curvature $(K)$ : each point $p$ in then represented by a vector $(u(p) ; S(p) ; K(p))$. This representation is displayed in Figure 4.

To improve robustness, and avoid that errors in segmentation or unwanted leaf artefacts (cracks, holes, spots) are taken into account, we want to keep only points that can be found on both sides of the leaf. Here again, the contour partition is very useful to know on which side lies a given point, and where to look on the contour to know if a similar one exists on the opposite side. The matching is performed by computing a distance term to all the points on the opposite side of same curvature sign, that takes into account their scale, curvature, relative position on the axis $[\hat{B} \hat{A}]$, and relative distance to this same axis. This method is somewhat risky but ensures that eccentric structures as in Figure 5 do not bias the understanding of the margin.
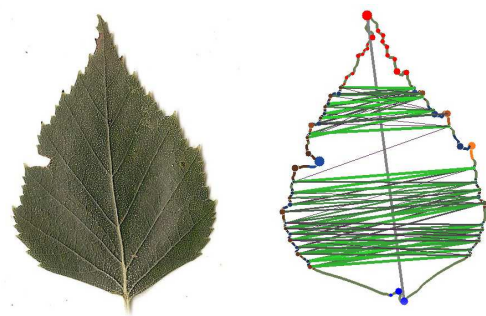

Figure 5: Suppression of points that can not be matched on a deteriorated leaf ; only connected points are kept

\subsection{Describing the Margin}

Even if this explicit representation is full of information and could be used as such to compare two leaves, it is still too heavy if we want to average several of them for classification purposes. It is necessary to sum it up into a condensed descriptor to capture the main properties of the margin, and allow a semantic interpretation of its specificities.

A first characterization consists in measuring how much of the leaf margin is convex, concave or neither of the two. Since the detected structures, given their definition, account for a number of vertices equal to their scale, we just have to sum the scales of dominant points and normalize them, to compute 3 parameters $w_{+}, w_{-}$and $w_{0}$, corresponding to the percentage of the set $\mathcal{M}$ of margin vertices that belong respectively to convex structures, concave structures, and no structure. 
To describe teeth properties in a condensed way, we compute averages and standard deviations for the properties we extracted on each dominant point. That gives 8 parameters computed on either concave (-) or convex (+) points, respectively $\bar{S}_{+}, \sigma_{S+}, \bar{S}_{-}, \sigma_{S-}$ for scale, and $\bar{K}_{+}, \sigma_{K+}, \bar{K}_{-}, \sigma_{K-}$ for curvature. These values are computed by weighting the considered parameter of each point by the point's scale, so that each vertex on the margin contributes at the same level.

Even if computing standard deviations provide a measure for the variability of the properties, such aggregating representation constitute a loss of spatial information. To keep a trace of the repartition of teeth along the margin, we computed the relative distances $d \uparrow(p)$ and $d \downarrow(p)$ of each point $p$, respectively to the next and previous points of opposite curvature on the same side $\mathcal{M}(p)$, when they exist.

These distances are once again averaged over points of the same curvature sign to produce 4 additional parameters $\overline{d \uparrow}_{+}, \overline{d \downarrow}_{+}$for convex points, and $\overline{d \uparrow}_{-}, \bar{d}_{\downarrow}$ for concave points, that account for the spatial repartition of teeth on the margin. What we finally use to describe the margin is a vector $P$ of 11 parameters :

$$
\begin{array}{lll}
\bullet \bar{S}_{+}+\sigma_{S+} & \bullet \bar{S}_{-}+\sigma_{S-} & \bullet w_{0} \\
\text { - } \bar{S}_{+}-\sigma_{S+} & \bullet \bar{S}_{-}-\sigma_{S-} & \bullet \overline{d \uparrow} / \overline{d \downarrow} \\
\bullet \bar{K}_{+}+\sigma_{K+} & \bullet \bar{K}_{-}+\sigma_{K-} & \bullet \overline{d \uparrow} / \overline{d \downarrow} \\
\bullet \bar{K}_{+}-\sigma_{K+} & \bullet \bar{K}_{-}-\sigma_{K-} &
\end{array}
$$

Taken together, these parameters constitute a very condensed yet efficient representation of what is important on a leaf contour, summing up the properties a botanist would investigate to characterize the margin. Most of the computational time comes from the CSS transform, but can be greatly reduced by computing only a limited number of scales and interpolating the curvature value for intermediate scales, leading to an execution time below the second on a computer processor, and of nearly 3 seconds on an iPhone 4 processor.

\section{CLASSIFICATION \& RESULTS}

To assess the relevance of our margin descriptors, we chose to classify leaves in botanical terms. However, it is impossible to have a database of leaves labelled with their exact shapes because of the intra-species variability and the subjectivity and vagueness of those words. The only way to learn automatically these shapes was to train a semi-supervised classifier using the possible shapes for each species and let it infer the concept represented by the classes.

\subsection{Learning Margin Shapes}

As a matter of fact, trying to evaluate the concepts behind the botanical words without exactly labelled examples is a very challenging issue. Those words maybe used to cover different shapes that may share some properties but not all of them. The method we used to learn these concepts tries to consider both the theoretical knowledge on leaf shapes and the uncertainty on the correspondence of one given leaf to one particular class.

Each considered species $s$ was labelled with one or more possible margin shapes $M(s)$, according to a reference botanical description (Coste, 1906). We retained 12 terms that were applicable to all the species we considered, namely :
- Entire

- Denticulate

- Undulate

- Crenate

- Serrate

- Dentate
- Doubly Serrate

- Sinuate

- Spiny

- Angular

- Lobate ${ }^{M}$

- Pinnatifid N
Then we used a semi-supervised Fuzzy C Means (FCM) clustering algorithm to learn the 12 centroids representing the shapes. Each individual $i$ in the database of species $s(i)$ is represented by a descriptor vector $P_{i}=\left(P_{k, i}\right)_{k=1 \ldots K}$ and is assigned a membership value $\left(\mu_{m, i}\right)_{m=1 . .12}$ representing its degree of belonging to each of the clusters. The supervising here consists of a constraint that the membership of an individual to clusters corresponding to shapes outside of the possible shapes $M(s(i))$ of its species must remain zero: $\forall i, \forall m=1 . .12, m \notin M(s(i)) \Longrightarrow \mu_{m, i}=0$. The initial membership values are set to be the same for each possible shape of the species, and the centroids are then computed following the regular FCM procedure, with a parameter $\beta$ set to 1.8 .

Each class is finally represented by its resulting centroid $C_{m}$ and by the estimated standard deviation vector $\Sigma_{m}$, computed using the memberships of each individual. A new leaf, represented by its margin parameter vector $P=\left(P_{k}\right)$ is then classified by computing its normalized Euclidean distance to all the class centroids, and assigning it to the closest.

\subsection{Species Identification}

The database used to test our algorithms is a subset of the Pl@ntLeaves (Goëau et al., 2011a) database, keeping only 5668 leaf images (out of 8422) on white, plain or natural backgrounds of 80 species (out of 126) with non-compound leaves. 
Parameters extracted on the images, outside of the margin descriptors described here [CS], are parameters from the polygonal leaf model [MP] (Cerutti et al., 2011) accounting for the global shape of the leaf, and parameters of Bezier curves based basal and apical shape models [B/A] estimated around the located points $\hat{B}$ and $\hat{A}$.

The data formed by all the parameters from all the images is first centered and normalized. Assuming that each parameter simply follows a Gaussian distribution, we compute class centroids and standard deviations with examples from the training dataset. For each species $s$, and for each possible number of lobes $n_{L}$ up to 3 we build a class $\Phi_{s, n_{L}}=$ $\left(\mu_{s, n_{L}, k}, \sigma_{s, n_{L}, k}\right)$ if at least $10 \%$ of all the species examples have this value.

To classify a new example, we will simply have to compare its descriptors with the centroids of the classes sharing the same number of lobes $n_{L}$. For each one of the 3 sets of parameters, we compute a Euclidean distance to the surface of the ellipsoid defined by means and standard deviations :

$$
\begin{aligned}
& \mathcal{D}\left(P, \Phi_{S, n_{L}}\right)=\left\|P-\mu_{\mathcal{S}, n_{L}}\right\|_{2} \max \left(1-\frac{1}{\left\|P-\mu_{S, n_{L}}\right\|_{M}}, 0\right) \\
& \left\|P-\mu_{S, n_{L}}\right\|_{M}=\sqrt{\sum_{k} \frac{\left(P_{k}-\mu_{s, n_{L}, k}\right)^{2}}{\sigma_{S, n_{L}, k}{ }^{2}}}
\end{aligned}
$$

Each one of these terms is then weighted differently after having learned the classes, dividing it by the average distance of the correct class over the training base, which weights it accordingly to its significance. The final distance we use for classification is simply the sum of these weighted terms, and the classes are then ordered accordingly to their distance, producing an ranked list of species.

\subsection{Experimental Results}

To evaluate the classification into botanical shapes, we performed a cross validation on the training base, learning the classes by FCM on two thirds of the database, and classifying the remaining third. A classification is assessed to be correct if the returned shape is one of the possible shapes taken by the leaf species. Results show a correct classification in $65 \%$ of cases, climbing up to $76 \%$ if we take into account the two first ranked classes.

We tried to refine this evaluation by building a confusion matrix for the shapes. It is not an easy task since the examples are not labelled by their actual shape, but by their species and thus their possible shapes. We decided to put a weight of 1 per example in the matrix, placing it in the diagonal cell if the classification was considered as correct, and splitting it among the theoretical possible classes in the recognized class column if not. The percentages are then obtained by dividing those weights by the total weight of each theoretical class.

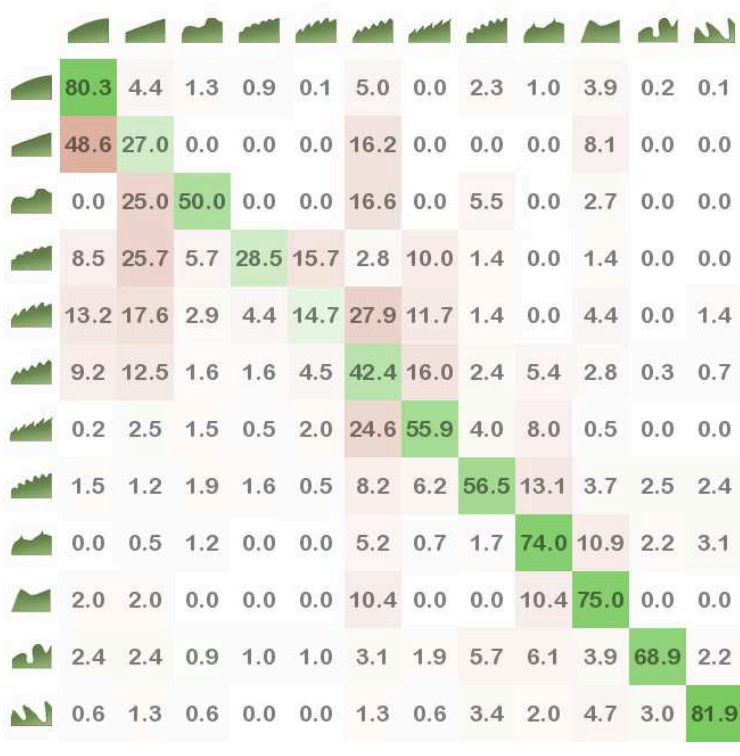

Table 1: Confusion Matrix for Leaf Margin Classification

The matrix we obtain is displayed in Table 1 and provides a good light on what our descriptors are good at discriminating or not. It appears clearly that margins with larger structures are easier to differentiate than smaller ones. It is difficult to capture the differences between for instance Serrate and Dentate leaves especially since the small scale we are looking at makes errors more common. Dentate is visibly a very variable class, making it more easily detected given the classification distance we use. Very small teeth of Denticulate leaves seem also hard to represent, such leaves being regularly seen as without teeth or with regular ones.

Concerning the species classification, results were evaluated following the same cross validation process. We computed classification rates for the different types of images in the Pl@ntLeaves Database (Scan, Pseudoscan and Photograph) and measured the presence of the true species among the top $\mathrm{k}$ answers ( $\mathrm{k}$ going from 1 to 10 ) returned by the classification algorithm.

Figure 6 presents the results obtained with the global, base and apex shape descriptors only $[\mathrm{MP}+\mathrm{B} / \mathrm{A}]$ and with the addition of the margin descriptors $[\mathrm{MP}+\mathrm{B} / \mathrm{A}+\mathrm{CS}]$, underlining the interest of considering the contour in a very specific way, with an average recognition score of $53 \%$, and a presence of the correct species in the 5 first answers of $85 \%$. The great improvement induced by the use 


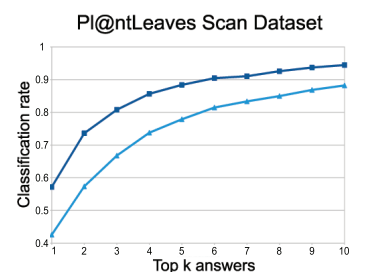

(a)

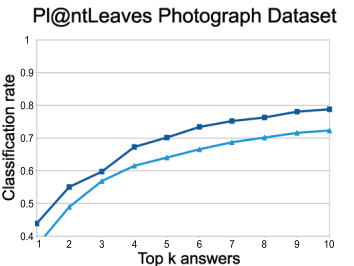

(c)

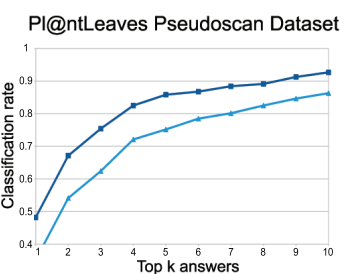

(b)

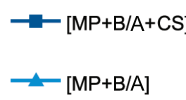

: Classification rates on the P1@ntLeaves Databa on scan (a) pseudoscan (b) and photograph (c) images

of curvature-based descriptors is here clearly visible. The enhancement is less important on photographs given the hardness of the task of retrieving the exact leaf contour, which obviously deteriorates the relevance of the contour description.

These scores may be compared to the results of the 2011 ImageCLEF Plant Identification task (Goëau et al., 2011a) concerning a very similar database, and where the best participants reached an average score of $45 \%$.

\section{CONCLUSION}

The method presented in this article constitutes an interesting step towards an explicative system of leaf classification for an independent mobile application. Being able to place high-level semantic concepts over the values extracted from an image gives the user a feedback that may prove of great usefulness for both interactive and educational purposes.

A first implementation of the recognition process on mobile devices is engaged with very satisfactory results, but not yet with the nearly 150 native European tree species we aim at recognizing. With the number of potential classes increasing, it will become a necessity to reduce the scope of the search, by including geographical information linked to the GPS system present in every smartphone. Knowing in advance which species are likely to be found in the geographical area where the user stands may be a decisive step towards a truly reliable identification.

However, what we have now is a good base structure for a new tree identification application, designed to be functional in a natural environment, and destined to anyone with interest in plants but without the otherwise compulsory botanical background.

\section{REFERENCES}

Belhumeur, P., Chen, D., Feiner, S., Jacobs, D., Kress, W., Ling, H., Lopez, I., Ramamoorthi, R., Sheorey, S., White, S., and Zhang, L. (2008). Searching the world's herbaria: A system for visual identication of plant species. In $E C C V$.

Caballero, C. and Aranda, M. C. (2010). Plant species identification using leaf image retrieval. In Proceedings of the ACM International Conference on Image and Video Retrieval, CIVR 2010, pages 327334.

Cerutti, G., Tougne, L., Mille, J., Vacavant, A., and Coquin, D. (2011). Guiding active contours for tree leaf segmentation and identification. In CLEF Working Notes.

Coste, H. (1906). Flore descriptive et illustrée de la France de la Corse et des contrées limitrophes.

Du, J. X., Wang, X. F., and Zhang, G.-J. (2007). Leaf shape based plant species recognition. Applied Mathematics and Computation, 185:883-893.

Goëau, H., Bonnet, P., Joly, A., Boujemaa, N., Barthelemy, D., Molino, J.-F., Birnbaum, P., Mouysset, E., and Picard, M. (2011a). The clef 2011 plant images classification task. In CLEF Working Notes.

Goëau, H., Joly, A., Yahiaoui, I., Bonnet, P., and Mouysset, E. (2011b). Participation of inria \& pl@ ntnet to imageclef 2011 plant images classification task. In CLEF Working Notes.

Im, C., Nishida, H., and Kunii, T. L. (1998). A hierarchical method of recognizing plant species by leaf shapes. In MVA, pages $158-161$.

Mokhtarian, F. and Abbasi, S. (2004). Matching shapes with self-intersections: Application to leaf classification. IEEE IP , 13(5):653-661.

Mokhtarian, F. and Mackworth, A. (1992). A theory of multiscale, curvature-based shape representation for planar curves. IEEE PAMI, 14:789-805.

Pei, S. C. and Lin, C. N. (1992). The detection of dominant points on digital curves by scale-space filtering. Pattern Recognition, 25(11):1307-1314.

Teh, C. H. and Chin, R. T. (1989). On the detection of dominant points on digital curves. IEEE PAMI, 11(8):859-872.

Teng, C.-H., Kuo, Y.-T., and Chen, Y.-S. (2009). Leaf segmentation, its $3 \mathrm{~d}$ position estimation and leaf classification from a few images with very close viewpoints. In ICIAR, pages 937-946.

Wang, X. F., Huang, D. S., Du, J. X., Huan, X., and Heutte, L. (2008). Classification of plant leaf images with complicated background. Applied Mathematics and Computation, 205(2):916-926.

Wang, Z., Chi, Z., Feng, D., and Wang, Q. (2000). Leaf image retrieval with shape features. In Advances in Visual Information Systems, volume 1929 of Lecture Notes in Computer Science, pages 41-52. 